\title{
Suicide rate following attendance at an accident and emergency department with deliberate self
} harm

\author{
J Ryan, A Rushdy, C A Perez-Avila, R Allison
}

\begin{abstract}
Objective-To determine the risk of suicide in patients attending an accident and emergency (A\&E) department with deliberate self harm.

Methods-Information was obtained on suicides and open verdicts from the coroner's office and cross checked with computerised records in the A\&E department. Results-There was a trend to suicide among younger socially isolated males and older females.

Conclusions-There is a significant association between suicide and a previous attendance at $A \& E$ with deliberate self harm. Appropriate assessment of these patients is an efficient way of managing self harm.

(F Accid Emerg Med 1996;13:101-104)
\end{abstract}

Key terms: deliberate self harm; suicide rate; accident and emergency

A history of deliberate self harm is known to increase the risk of suicide by 10 - to 30 -fold and this risk is greatest in the year after parasuicide. ${ }^{12}$ In view of this strong association between deliberate self harm and suicide, we assessed the subsequent suicide rate of patients who attended the accident and emergency (A\&E) department of the Royal Sussex County Hospital (RSCH) with deliberate self harm. We also investigated the role of the $A \& E$ department in the proposed reduction in overall suicide rates of $15 \%$ set in The health of the nation White Paper. ${ }^{3}$

Accident and
Emergency
Department,
Royal Sussex County
Hospital, Eastern
Road, Brighton
J Ryan
C A Perez-Avila
Department of Public
Health Medicine,
Regency House,
95 Ditchling Road,
Brighton
A Rushdy
Department of
Psychiatry
Community Mental
Health Centre,
35 New Church Rd,
Hove
R Allison
Correspondence to:
Mr John M Ryan,
Accident and Emergency
Department, King's College
Hospital, Denmark Hill,
London SE5 9RS,
United Kingdom.

\section{Methods}

The A\&E department of the RSCH sees 54000 new patients a year and serves a population of 300000 people. The incidence of patients with deliberate self harm attending the department is $3.4 \%$ of all attendances. In our hospital the term "deliberate self harm" means deliberate self poisoning or deliberate self injury and is synonymous with parasuicide and attempted suicide.

Patients who attend our A\&E department with deliberate self harm are assessed by the A\&E doctor using the modified SAD PERSONS score, which is a 10 letter mnemonic representing risk factors for suicidal patients (table 1). The score is used as an objective tool to assess the risk of suicide. New senior house officers and registrars receive training in its use from liaison psychiatry staff during their first week in the department.
Many self harm patients with complicated psychosocial or physical problems are admitted to the A\&E observation ward. They are assessed the following morning by a multidisciplinary team which is medically led by the A\&E consultant or senior registrar and includes a social worker, psychiatric liaison nurse, community liaison nurse, physiotherapist, and ward sister. The team assesses the patient's physical, social, and psychological condition. A psychiatric review is requested if the patient is felt to be at risk of suicide.

We consulted the coroner for the Brighton, Hove, and Lewes areas for which the RSCH is the main acute hospital. He supplied us with the names of people who had committed suicide or who had an open verdict returned at their inquest during 1992 and 1993. As with other studies we have regarded open verdicts as suicides. ${ }^{5}$ We included people resident in our area who had committed suicide elsewhere. We obtained dates of death as well as some demographic details, including the patient's age, sex, employment situation, and marital status. We investigated if there was a past history of psychiatric problems or previous suicide attempts, and the method of suicide.

The A\&E department has been computerised since 1987. We consulted A\&E department records to see how many of the 1992 and 1993 suicides attended the $A \& E$ department in the previous seven years. We reviewed these notes with particular reference to previous presenting complaints and management.

\section{Results}

During the two year period reviewed we recorded 3683 attendances with episodes of deliberate self harm. Almost half were managed in the $A \& E$ department by $A \& E$ staff and were either discharged, admitted to the general wards, or took their own discharge before they could be dealt with appropriately (table 2). A few were seen by the duty psychiatrist in the $A \& E$ department. One third were admitted to the A\&E observation ward and were managed by an $A \& E$ based multidisciplinary team.

Ninety eight patients committed suicide or had an open verdict returned by the coroner during the two years reviewed. We found records of $A \& E$ attendances for 40 patients. Seven of these presented to the A\&E department with what proved to be a fatal deliberate self harm episode. They were not previously known to the department and have been excluded from subsequent analysis. 
Table 1 Modified SAD PERSONS score

$\mathrm{S}=\mathrm{Sex}$

$A=$ Age $\quad<19$ or $>45$

$\mathrm{D}=$ Depression or hopelessness Admits to depression or decreased concentration, appetite or sleep.

or decreased concentration,

$P=$ Previous attempts or psychiatric care

$\mathrm{E}=$ Excessive alcohol or drug use

$\mathrm{R}=$ Rational thinking loss

$S=$ Separated, divorced or widowed

$\mathrm{O}=$ Organised or serious attempt

Stigmata of chronic addiction or recent frequent use. Organic brain syndrome or psychosis.

$\mathrm{N}=$ No social supports

$\mathrm{S}=$ Stated future intent

Well thought out plan or life threatening presentation.

No close family, friends, job, or active religious affiliation

Each risk factor if present scores one point. A score of $<3$ is taken as low risk, 3-6 as medium risk, and $>6$ as high risk.

Of the 33 patients who had attended A\&E on a previous occasion and later went on to commit suicide, 23 were male and 10 were female. The median age at time of death was between 21 and 25 years of age. The mean age at time of death was 40.7 years ( 54.6 years for females and 34.7 years for males) (figure).

On average, patients last attended the $A \& E$ department five months before dying (range 5 days to 15 months, median 1 month.)

Most patients attended the $\mathrm{A} \& \mathrm{E}$ department just once before their death (18 patients) but the range was from one to 29 visits.

Eleven patients previously attended with episodes not classified as deliberate self harm. Four presented following an assault, four with acute exacerbation of a chronic illness, one with a facial laceration, one with a suspected deep vein thrombosis, and one with a penile injury. In retrospect, one patient's foot injury, sustained by a gunshot injury, and another patient's left arm wound may have been self inflicted.

In 1992 and 1993 we admitted $1 \cdot 7$ (1203/698) times as many cases of deliberate

Table 2 Outcome of patients presenting with deliberate self harm over 2 years

\begin{tabular}{lcl}
\hline & $\begin{array}{l}\text { Number } \\
(\%)\end{array}$ & $\begin{array}{l}\text { Subsequent } \\
\text { suicide rate }\end{array}$ \\
\hline Discharged from A\&E & $1738(47 \%)$ & $0.69 \%$ \\
Admitted to observation ward & $1203(33 \%)$ & $0.83 \%$ \\
Admitted Med/Surg/ITU & $698(19 \%)$ & $1.1 \%$ \\
Admitted by psychiatrist & $44(1 \cdot 2 \%)$ & $0.00 \%$ \\
Total & $3683(100 \%)$ & $1.08 \%$ \\
(Number who commited suicide & 33 & $0.9 \%)$ \\
\hline
\end{tabular}

Med, medical ward; Surg, surgical ward; ITU, intensive care unit.

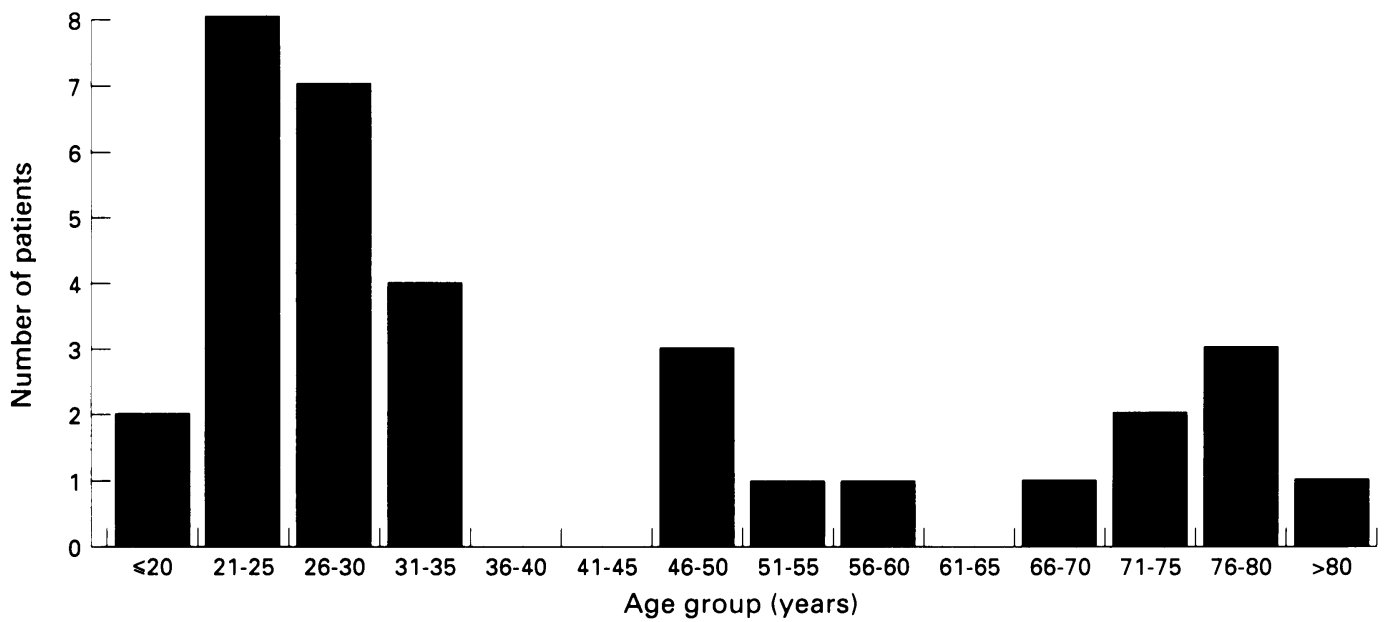

Age range of patients committing suicide self harm as were admitted to the general wards. The suicide rate for patients admitted to the observation ward following deliberate self harm was lower $(0.83 \%$ of admissions) compared with those admitted to the general wards ( $1.1 \%$ of admissions)

Twenty one patients attended the A\&E department previously with clearly identifiable episodes of deliberate self harm. Twelve of these 21 patients were discharged from the $A \& E$ department, admitted to the general wards, or took their own discharge. Nine were admitted to the observation ward and had a multidisciplinary assessment and calculation of the SAD PERSONS score. In addition to these nine patients, one elderly patient was admitted to the observation ward after a fall and discharged the following day. She was readmitted one week later with a probable quinine overdose which was to be fatal. We detail these cases below.

CASE 1

A 49 year old separated man presented to the A\&E department having cut his wrists. He had recently separated from his wife and had financial difficulties. He had a history of one overdose some 10 years previously. His SAD PERSONS score was 6 . He was seen by a psychiatrist and discharged from the observation ward. Follow up was arranged with a community psychiatric nurse. He did not attend for follow up. Ten weeks later he committed suicide by carbon monoxide poisoning.

\section{CASE 2}

A 24 year old unemployed single man was admitted following an overdose. He had a long history of depression and was well known to local psychiatric services. He had attended the $A \& E$ department on many occasions with episodes of deliberate self harm. His SAD PERSONS score was 5 . He died from self inflicted burns three months after his discharge.

CASE 3

A 28 year old single man who was well known to psychiatric services was admitted following an overdose. He had a long history of depression and self harm. His SAD PERSONS score 
was 4. He was transferred to a local psychiatric hospital following a psychiatric evaluation. Eight months later he was found hanging.

CASE 4

A 27 year old unemployed single man with a history of depression and previous overdoses was admitted following a mixed overdose. His sad PERSONS SCORE was 4. He committed suicide just over two months after discharge by asphyxiation.

CASE 5

A 97 year old widow was admitted following a fall in her warden-supervised accommodation. She had attempted suicide at home two months previously. She was subsequently seen by a psychiatrist and diagnosed as suffering from agitated depression. This previous psychiatric history was not known to $A \& E$ staff at the time of her admission to the observation ward. One week after her discharge she was brought back to the A\&E department having been found moribund at home. She died shortly afterwards. Toxic levels of quinine were found at necropsy.

CASE 6

A 30 year old landscape gardener presented to the A\&E department on 29 occasions over a period of five years, mostly with episodes of deliberate self harm. He was well known to local psychiatric services. He was admitted with self inflicted lacerations and an overdose of nitrazepam. He had recently separated from his partner. He was seen by the duty psychiatrist in the A\&E department. His SAD PERSONS score was 5 . He was found dead three weeks following his discharge.

CASE 7

A 35 year old unemployed HIV positive drug addict was admitted following an overdose. His SAD PERSONS score was 8 . He was well known to psychiatric services and to the A\&E department because of previous overdoses. He was transferred to a psychiatric hospital the day after admission. Three months later he was found dead.

CASE 8

A 27 year old married schizophrenic man with a long psychiatric history was admitted following a mixed overdose. His SAD PERSONS score was 4 . He was admitted to the hospital with serious overdoses over the following three years and was ultimately brought to the $A \& E$ department in cardiac arrest having been rescued from the sea.

CASE 9

A 21 year old unemployed single woman with a long psychiatric history was admitted following an overdose of alcohol and benzodiazepines. Her SAD PERSONS score was 4. She had 17 previous attendances at the A\&E department. She was found dead two years after discharge from the ward, although she continued to attend the $\mathrm{A} \& \mathrm{E}$ department during this time.
CASE 10

A 70 year old woman was admitted with hypothermia having been found sitting on the roadside in the middle of winter. She self discharged from the ward the following day. Three weeks later she was found dead.

\section{Discussion}

This study confirms the significant association between presentation to an A\&E department with an episode of deliberate self harm and subsequent suicide. Patients with a previous history of deliberate self harm were 18 times more likely to kill themselves than members of the local population (300 000).

The subsequent suicides of deliberate self harm patients discharged from an observation ward has not been reported before now, although it has been shown in the past that patients sent home from an A\&E department following deliberate self harm are screened adequately by $A \& E$ staff. ${ }^{6}$ In line with other studies, we had a high rate of discharge from $A \& E$ for patients presenting with deliberate self harm $(47 \%){ }^{7-10}$ The A\&E observation ward has been found to be an effective way of managing overdose patients. ${ }^{11}$ We feel that an $A \& E$ observation ward is an appropriate place to admit self harm patients who require observation or brief medical intervention, or to defuse a social crisis.

We have identified four groups of patients attending the $A \& E$ department who later committed suicide or had an open verdict returned on their death.

(1) Eleven patients attended the A\&E department with an episode unrelated to deliberate self harm. Thus other than maintaining a general awareness of mental health issues there is little else that we can do to identify these patients as being at risk at the time of presentation.

(2) Seven patients attended the A\&E department with a clear episode of deliberate self harm but were not admitted. Two of these had fractures of the 5th metacarpal sustained when they punched a wall. We now counsel people who present with boxer's fractures and are vigilant when dealing with patients who present with injuries which could possibly be self inflicted.

(3) Ten patients were admitted to the A\&E observation ward and managed by the A\&E multidisciplinary team. The details of this group have been outlined in the case histories above. Of concern is the frequency of suicide in younger males.

(4) Five patients were admitted to the general wards following serious episodes of deliberate self harm.

We have found the SAD PERSONS score a useful tool in assessing the suicide risk of patients admitted to our ward with deliberate self harm. On review of the SAD PERSONS scores of the 10 patients admitted to the observation ward we found that the two patients with the highest scores were referred to the on-call psychiatrist and one was admitted to a psychiatric ward. A psychiatric opinion had also been requested on the one patient who died within 
two months of being a patient on the observation ward. All patients had a score of at least 4 , reflecting at least a medium risk. We suggest that an objective scoring system such as the SAD PERSONS score, admission to an A\&E observation ward, and a multidisciplinary team review is a pertinent way of managing patients who present to the $A \& E$ department with episodes of deliberate self harm requiring admission who do not need significant medical or surgical input.

We recognise that the $A \& E$ department has a significant role to play in treating patients with deliberate self harm and has the potential to prevent some suicides. It is undesirable that any patient who has been through the system with an episode of deliberate self harm should later commit suicide, but following review of their records we have been unable to see what further action we could have taken to prevent their subsequent suicide. It is difficult to see how much more the A\&E department can contribute to reducing the rate of suicide by the $15 \%$ recommended by the Health of the nation White Paper. We wonder if recent attendance at an $A \& E$ department with deliberate self harm should be considered as a predictor of suicide and whether more assertive community outreach from the mental health service could improve the outcome in the high risk group.

1 Hockberger RS, Rothstein RJ. Assessment of suicide Hockberger RS, Rothstein RJ. Assessment of suicide potential by non psychiatrists using
score. $\mathcal{F}$ Emerg Med 1988;6:99-107.

2 Hawton K, Fagg J. Suicide, and other causes of death, following attempted suicide. $B r \quad \mathcal{F}$ Psychiatry 1988; 152:359-66

3 Nordentoft $M$, Breum L, Munck L, Nordestgaard A, Hunding A, Bjaeldager P. High mortality by natural and unnatural causes: a 10 year follow up study of patients admitted to a poisoning treatment centre after suicide attempts. $B M F$ 1993;306:1637-40.

4 Secretary of State for Health. The health of the nation: $a$ strategy for health in England. London: HMSO, 1992.

5 Adelstein A, Mardon C. Suicides 1961-74. In: Population trends vol 2. London: HMSO, 1975:13-18.

6 Gardner $R$, Hanka $R$, Roberts SJ, Allon-Smith JM, Kings AA, Nicholson R. Psychological and social Kings AA, Nicholson $R$. Psychological and social accident department. BMF 1982;284:491-3.

7 Greer S, Bagley C. (1971) Effect of psychiatric intervention in attempted suicide: a controlled study. $B M \mathcal{F} 1971$; i:310-12.

8 Lintner CM. Psychiatric involvement in cases of deliberate self harm. Br F Soc Clin Psychiatry 1985;3:11-15.

9 Owens DW, Jones SJ. The accident and emergency department management of deliberate self-poisoning. $\mathrm{Br} f$ Psychiatry 1988;152:830-3.

10 Owens D. Self-harm patients not admitted to hospital. $\mathcal{F} R$ Coll Physicians Lond 1990;24:281-3.

11 Driscoll P, Bryce G. The use of short-stay wards. A survey of 1,000 admissions. Health Bull 1987;45/6:294-302.

For further information contact: Ms Sally Jenner, Association of Anaesthetists of Great Britain and Ireland, 9 Bedford Square, London WC1B 3RA. (Tel 0171631 1650.) 